\title{
COMUNICAÇÃOCIENTÍFICA
}

\section{PROPAGAÇÃO DA FIGUEIRA 'ROXO DE VALINHOS' POR ALPORQUIA ${ }^{1}$}

\author{
SIMONE DANELUZ ${ }^{2}$, RAFAEL PIO ${ }^{3}$, EDVANALVES CHAGAS ${ }^{4}$, \\ WILSON BARBOSA ${ }^{5}$, TATIANE OHLAND ${ }^{2}$, TAILENE ELISA KOTZ $^{2}$
}

RESUMO - O objetivo do presente trabalho foi estudar a posição do ramo associada a diferentes ferimentos, substratos e concentrações de ácido indolbutírico (AIB) no enraizamento de alporques de figueira 'Roxo de Valinhos'. No primeiro experimento, foram realizados alporques na porção basal, mediana e apical dos ramos, no início do mês de março. No preparo dos alporques, foram realizados diferentes ferimentos: anel inteiro (anéis de três $\mathrm{cm}$ de comprimento), dois cortes (cortes paralelos de três $\mathrm{cm}$ de comprimento e um $\mathrm{cm}$ de largura), um corte (corte de três $\mathrm{cm}$ de comprimento e um $\mathrm{cm}$ de largura) e a testemunha com ausência de corte. Em seguida, colocou-se, no local do tratamento, substrato à base de casca de pínus umedecido, envolvendo-se com plástico transparente e amarrando-se nas extremidades, para evitar a perda de umidade. Passados 50 dias, os alporques foram removidos para a avaliação da porcentagem de alporques calejados, enraizados e o número médio de raízes. Em seguida, foram transplantados para leito de areia umedecido sob telado constituído de sombrite com 50\% de luminosidade. Passados 30 dias, foram mensurados a porcentagem de alporques vivos e brotados, e o número médio de brotos. No segundo experimento, os alporques foram realizados no mês de abril, na porção mediana dos ramos, sem ferimento, aplicando-se no local, diferentes concentrações de AIB $\left(0 ; 1.000 ; 2.000\right.$ e $\left.3.000 \mathrm{mg} \mathrm{L}^{-1}\right)$ e, em seguida, foram envolvidos pelos seguintes substratos: casca de pínus umedecido, esfagno umedecido e a mistura de ambos na proporção $1: 1 \mathrm{v} / \mathrm{v}$. Passados 60 dias, os alporques foram removidos para a mensuração da porcentagem de alporques calejados, enraizados e o número médio de raízes. Concluiu-se que os alporques devem ser realizados na porção mediana dos ramos, ausentes de ferimento, tratados com $1.000 \mathrm{mg} \mathrm{L}^{-1}$ de AIB e envolvidos com substrato à base de casca de pínus.

Termos para Indexação: Ficus carica L., estaquia e ácido indolbutírico.

\section{PROPAGATION OF THE FIG TREE ‘Roxo de Valinhos’ BY AIR LAYERING}

ABSTRACT - The objective of the present work was to study the position of the branch associated to different wounds, substrates and concentrations of indolbutyric acid (IBA) in the rooting of air layering in 'Roxo de Valinhos' fig tree. In the first experiment, air layering was accomplished in the basal, medium portion and apical of the branches in the beginning of the month of March. In the prepare the air layering, different wounds were accomplished: whole ring (rings of three $\mathrm{cm}$ of length), two cuts (parallel cuts of three $\mathrm{cm}$ of length and a $\mathrm{cm}$ of width), a cut (measuring three $\mathrm{cm}$ of length and one $\mathrm{cm}$ of width) and the witness cut absence. Soon after, it was placed in the place of the treatment, substrate of humidified pinus, wrapped up with transparent plastic and being tied in the extremities, to avoid the humidity loss. After 50 days, the air layering was removed for the evaluation of the percentage of case-hardened and rooted layers and the medium number of roots. Soon after, they were transplanted for bed of sand humidified under nursery constituted of sombrite with $50 \%$ of brightness. After 30 days, it was evaluated the percentage of alive, air layering sprouted and the medium number of sprouts. In the second experiment, the air layering was accomplished in the month of April, in the medium portion of the branches, without wound, being applied in the place, different concentrations of $\operatorname{IBA}\left(0,1000,2000\right.$ and $\left.3000 \mathrm{mg} \mathrm{L}^{-1}\right)$ and soon after, they were involved by the following substrate: humidified pinus, humidified sphagnum and the mixture in the proportion $1: 1 \mathrm{v} /$ v. After 60 days, the air layering was removed for the evaluated of the percentage of case-hardened and rooted layers and the medium number of roots. It was concluded that the air layering should be accomplished in the medium portion of the branches, absent of wound, agreements with $1000 \mathrm{mg} \mathrm{L}^{-1}$ of IBA and involved with pinus substrate.

Index terms: Ficus carica L., cutting and indolbutyric acid.

\footnotetext{
${ }^{1}$ (Trabalho 072-08). Recebido em: 25-03-2008. Aceito para publicação em: 04-11-2008.

${ }^{2}$ Acadêmico do curso de Agronomia, Universidade Estadual do Oeste do Paraná - UNIOESTE. Rua Pernambuco, 1.777, Caixa Postal 1.008, Centro, 85960-000, Marechal Cândido Rondon-PR. simonedaneluz@yahoo.com.br tatianeohland@hotmail.com taileneelisa@hotmail.com

${ }^{3}$ Eng $^{\circ}$. Agrônomo, D.Sc., Professor Adjunto da Universidade Estadual do Oeste do Paraná - UNIOESTE. Rua Pernambuco, 1.777, Caixa Postal 1.008, Centro, 85960-000, Marechal Cândido Rondon-PR. Bolsista Produtividade em Pesquisa CNPq. Autor para correspondência: rafaelpio@ hotmail.com

${ }^{4} E_{n g}{ }^{\circ}$. Agrônomo, D.Sc., Pesquisador Científico Empresa Brasileira de Pesquisa Agropecuária-Embrapa Roraima. BR 174, km 8- Dist.Industrial, 69301-970, Boa Vista-RR. Bolsista Produtividade em Pesquisa CNPq. echagas@pq.cnpq.br

${ }^{5}$ Biólogo, M.Sc., Pesquisador Científico, Centro Experimental Central, Instituto Agronômico - IAC. Caixa Postal 28 , 13001-970, Campinas-SP. Bolsista Produtividade em Pesquisa CNPq. wbarbosa@iac.sp.gov.br
} 
O cultivo da figueira (Ficus carica L.) vem apresentando ligeira expansão mundial, frente as suas peculiaridades quanto à rusticidade e adaptabilidade às mais diversas condições climáticas, possibilidade em destinar a produção para o mercado de fruta fresca ou para o processamento industrial e, no caso do Brasil, exportação dos frutos na entressafra dos países produtores do Hemisfério Norte (Chalfun et al., 1997).

Quanto aos aspectos propagativos, a multiplicação da figueira é geralmente realizada através de estacas caulinares lenhosas, coletadas no momento da poda hibernal, efetuada entre os meses de junho e julho (Alvarenga et al., 2007). Os ficicultores brasileiros, normalmente, padronizam as estacas com $40 \mathrm{~cm}$ de comprimento e três $\mathrm{cm}$ de diâmetro, efetuando-se o plantio das estacas diretamente na cova. Essa prática vem acarretando elevado custo de implantação dos pomares, devido ao baixo índice de enraizamento das estacas, por não haver coincidência da estaquia com o período chuvoso, principalmente nas regiões Sul e Sudeste, originando desuniformidade na formação do figueiral e, muitas vezes, necessidade de replantio (Chalfun et al., 1997; Gonçalves et al., 2003).

A solução para a propagação da figueira seria o enraizamento prévio das estacas em ambiente protegido, podendo assim utilizar estacas de menor comprimento, facilitando o manejo das mudas no viveiro, além de propiciar a seleção de plantas de qualidade e plantio no período chuvoso, possibilitando a obtenção de um pomar uniforme e vigoroso. No entanto, os índices de enraizamento das estacas coletadas na época da poda hibernal da figueira não ultrapassam a 50\% (Norberto et al., 2001).

Baixo enraizamento dos propágulos vegetativos pode estar correlacionado a fatores intrínsecos ao material vegetal, como a idade do tecido, a época de coleta das estacas, a concentração de fitormônios ou ainda a fatores exógenos, como as condições de cultivo das estacas (Hartmann et al., 2002). Nesse âmbito, a adoção de outras metodologias para a propagação vegetativa da figueira, como a alporquia, técnica propagativa ainda não estudada para a frutífera em questão, no, Brasil, poderia ser uma alternativa viável economicamente para a produção de mudas de figo.

A alporquia concilia o enraizamento de uma porção do ramo ainda conectado à planta-matriz, melhorando as condições para que a rizogênese aconteça. Assim, o desenvolvimento das raízes é auxiliado pelo anelamento do ramo, impedindo que os carboidratos, hormônios e outras substâncias produzidas pelas folhas e gemas, necessárias à rizogênese, sejam translocadas para outras partes da planta. Por sua vez, o xilema não é afetado, fornecendo água e elementos minerais ao ramo (Hartmnn et al., 2002).

A propagação pelo método de alporquia apresenta vantagens em relação à estaquia, dentre as quais estão o alto percentual de enraizamento e a independência de infraestrutura (casa de vegetação com sistema de nebulização) (Castro \& Silveira, 2003).

No caso da figueira, em detrimento da fenologia da planta, os alporques devem ser realizados entre o final da safra e o início da dormência das plantas, concentrando-se entre os meses de março e abril, para assim não haver prejuízos na produção da planta, já que o ciclo produtivo se estende entre os meses de outubro e meados de março, nas condições das regiões Sul e Sudeste do Brasil e, ainda, aproveitar a retirada dos alporques no momento da poda hibernal, realizada entre os meses de junho e julho, já que, na condução da figueira, adota-se a poda drástica dos ramos que produziram no ciclo anterior (Chalfun et al., 2002).

O sucesso dessa metodologia de propagação está associado ao indício de melhorias no enraizamento, frente a este evento ocorrer com os ramos ainda ligados à planta-matriz, ao período ótimo de enraizamento, que, segundo a literatura, concentra-se nos meses que antecedem a poda hibernal (Norberto et al., 2001) e ainda pela possibilidade de levar uma muda ao campo com a primeira pernada já estabelecida, uma vez que a alporquia é realizada abaixo do ápice do ramo, podendo assim levar esse fragmento de caule enraizado em outubro ou novembro do mesmo ano, com até $30 \mathrm{~cm}$ de comprimento. Isso propiciaria, além do maior rendimento da formação das mudas, a antecipação da formação do pomar, visto que mudas oriundas de estacas coletadas em julho demandam dois meses para enraizar e, posteriormente, quatro a cinco meses para desenvolver a brotação e assim estar apta para plantio (Pio, 2002).

Assim, o objetivo do presente trabalho foi estudar a posição do ramo associada a diferentes ferimentos, bem como substratos e concentrações de ácido indolbutírico (AIB) no enraizamento de alporques de figueira 'Roxo de Valinhos'.

Foram realizados dois experimentos, em um pomar comercial de figueira 'Roxo de Valinhos', de quatro anos de idade, localizado no município de Quatro, Pontes-PR, entre os meses de março a junho e 2007.

No primeiro experimento, foram realizados alporques na porção basal, mediana e apical dos 
ramos, no início do mês de março. No preparo dos alporques, foram realizados diferentes ferimentos: anelamento inteiro (anelamento de três $\mathrm{cm}$ de comprimento), dois cortes (cortes paralelos de três $\mathrm{cm}$ de comprimento e um $\mathrm{cm}$ de largura), um corte (corte de três cm de comprimento e um cm de largura) e a testemunha com ausência de corte. Em seguida, colocou-se, no local do tratamento, substrato a base de casca de pínus umedecido, envolvendo-se a seguir com plástico transparente e amarrando-se nas extremidades, para evitar a perda de umidade. $\mathrm{O}$ delineamento utilizado foi o inteiramente casualizado, no esquema fatorial 3 (posição) x 4 (tipo de corte), com quatro repetições e cinco alporques por parcela. Passados 50 dias, os alporques foram removidos para a mensuração da porcentagem de alporques calejados, enraizados e o número médio de raízes. Em seguida, uma amostragem de três alporques por parcela, de cada repetição, foi transplantada para leito de areia umedecido, enterrando-se $2 / 3$ de seu comprimento, sob telado constituído de sombrite com $50 \%$ de luminosidade. Passados 30 dias, foram mensurados a porcentagem de alporques vivos e brotados, e o número médio de brotos.

No segundo experimento, os alporques foram realizados no mês de abril, na porção mediana dos ramos, sem ferimento, aplicando-se no local diferentes concentrações de AIB $(0 ; 1.000 ; 2.000$ e $3.000 \mathrm{mg} \mathrm{L}^{-1}$ ) e, em seguida, foram envolvidos pelos seguintes substratos: casca de pínus umedecido, esfagno umedecido e a mistura de ambos, na proporção 1:1 v/v. O delineamento utilizado foi o inteiramente casualizado, no esquema fatorial 4 (concentrações de AIB) x 3 (substratos), com quatro repetições e cinco alporques por parcela. Passados 60 dias, os alporques foram removidos para a mensuração da porcentagem de alporques calejados, enraizados e o número médio de raízes.

Os dados foram submetidos à análise de variância, as médias comparadas pelo teste Tukey, ao nível de $5 \%$ de probabilidade, e as concentrações de AIB submetidas à regressão, segundo as recomendações de Gomes (2000). As análises foram realizadas pelo programa computacional Sistema para Análise de Variância - SISVAR (Ferreira, 2000).

Segundo a análise estatística do primeiro experimento, não houve interação entre os fatores posição do ramo e ferimento (Tabela 1). No entanto, para a realização da alporquia em função da posição do ramo, alporques realizados na porção basal dos ramos apresentaram maior porcentagem de calejamento $(87,75 \%), 19 \%$ a mais que os alporques realizados na porção mediana e $37,11 \%$ a mais, em comparação aos realizados na porção apical (Tabela 1). Quanto ao enraizamento, não houve diferença entre os tratamentos, apesar de que, numericamente, alporques realizados na porção mediana apresentam melhor resultado $(53,75 \%)$. Já para o número médio de raízes, alporques realizados na porção apical apresentaram resultados superiores $(26,79)$.

Quanto à posição do ramo, o fato de alporques realizados na porção basal terem promovido maior porcentagem de formação de calos, pode estar associada à maior quantidade de reservas (carboidratos) contidas nesse fragmento de ramo (Fachinello et al., 2005). No entanto, conforme relata Pio et al. (2006b), estacas retiradas da porção basal dos ramos promovem menor enraizamento, quando comparadas às estacas retiradas das porções medianas e apicais. Quanto às estacas coletadas na porção apical dos ramos, Pio et al. (2006b) referem-se ao alto potencial rizogênico dos fragmentos coletados nessa posição dos ramos, principalmente no que tange ao número de raízes por estaca, quando obtiveram 15,37 raízes em média, igualmente ocorrido no experimento em pauta, porém obtiveram-se 11 raízes a mais, em média (Tabela 1).

Apesar dos bons resultados ocorridos com os alporques realizados na porção apical dos ramos, ressalta-se que, durante a condução do experimento, notou-se a quebra de alguns alporques, devido ao peso excessivo do alporque sobre a extremidade do ramo. Assim, quanto aos aspectos práticos, deve-se evitar a realização de alporques nessa porção dos ramos.

$\mathrm{Na}$ avaliação ocorrida 30 dias após o transplantio, houve $100 \%$ de sobrevivência em todos os tratamentos, porém alporques oriundos da porção mediana apresentaram alta porcentagem de brotação e número médio de brotos (Tabela 1).

Quanto ao ferimento no preparo dos alporques, alporques ausentes de ferimento e com apenas um corte apresentaram maior porcentagem de calejamento e enraizamento, não ocorrendo diferença estatística entre os tratamentos na mensuração do número de raízes por alporques (Tabela 2). Na avaliação ocorrida 30 dias após o transplantio, houve tendência da supremacia da realização de apenas um corte no preparo dos alporques, quanto à porcentagem de brotação e número médio de brotos (Tabela 2). Observou-se, no momento da remoção dos alporques dos ramos, que os tratamentos com anel e dois cortes apresentaram apodrecimento do local onde se realizou o ferimento, ocorrendo, em ambos os casos, enraizamento acima ou abaixo do ferimento, fato esse que proporcionou resultados inferiores, em comparação ao menor grau 
de ferimentos nos ramos alporqueados.

A análise estatística do segundo experimento revelou a não-interação entre os fatores substratos e AIB. Não houve diferença entre os substratos na mensuração da porcentagem de alporques calejados, ressaltando-se, no entanto, os bons resultados obtidos (acima de 97\%); quanto à porcentagem de enraizamento dos alporques e número médio de raízes, houve tendência de o substrato casca de pínus apresentar melhores resultados (Tabela 3). A superioridade do substrato casca de pínus pode estar relacionada à maior retenção de umidade, o que provavelmente favoreceu a rizogênese dos alporques, conforme relatam Smiderle \& Minami (2001).

Para a utilização do AIB, verifica-se, pelas Figuras 1 e 2, que a concentração de $1.000 \mathrm{mg} \mathrm{L}^{-1}$ do fitorregulador promoveu aumento significativo na porcentagem de alporques enraizados $(53,42 \%)$ e no número médio de raízes (seis raízes). Com a aplicação dessa concentração, conseguiu-se aumento de $22,2 \%$ de enraizamento dos alporques. Pio et al. (2004) também evidenciam aumento significativo com a aplicação de AIB, em relação à ausência de tratamento, no enraizamento de estacas de figueira. Segundo Fachinello et al. (2005), entre as principais funções biológicas das auxinas, pode-se citar o crescimento de órgãos, especialmente as raízes. Segundo Hartmann et al. (2002), é de extrema importância à utilização correta das concentrações de fitorreguladores a serem aplicados para promoverem o máximo enraizamento, sendo que a concentração ideal varia com a espécie em que se está trabalhando.

Através dos resultados obtidos, concluiuse que: a alporquia é uma metodologia viável para a produção de mudas de figueira; os alporques devem ser realizados na porção mediana dos ramos, confeccionados sem a adoção de quaisquer ferimentos; o substrato casca de pínus e a concentração de $1.000 \mathrm{mg} \mathrm{L}^{-1}$ de AIB proporcionaram os melhores resultados na rizogênese dos alporques.

TABELA 1- Porcentagem de alporques calejados, enraizados e número médio de raízes de alporques de figueira 'Roxo de Valinhos' (Ficus carica L.), realizados em diferentes posições no ramo, e porcentagem de alporques vivos, brotados e número médio de brotos após 30 dias do transplantio. Marechal Cândido Rondon-PR, UNIOESTE, 2007.

\begin{tabular}{|c|c|c|c|c|c|c|}
\hline \multirow{2}{*}{$\begin{array}{l}\text { Posição } \\
\text { no ramo }\end{array}$} & \multicolumn{3}{|c|}{ Variáveis analisadas aos 60 dias* } & \multicolumn{3}{|c|}{ Variáveis analisadas após transplantio* } \\
\hline & $\begin{array}{c}\% \text { Alporques } \\
\text { calejados }\end{array}$ & $\begin{array}{c}\text { \% Alporques } \\
\text { enraizados }\end{array}$ & $\begin{array}{l}\mathrm{N}^{\circ} \text { raízes } \\
\text { alporque }\end{array}$ & $\begin{array}{c}\% \text { Alporques } \\
\text { vivos }\end{array}$ & $\begin{array}{c}\% \text { Alporques } \\
\text { brotados }\end{array}$ & $\begin{array}{c}\mathrm{N}^{\circ} \text { médio } \\
\text { brotos }\end{array}$ \\
\hline Basal & 87,75 a & 42,50 a & $11,03 \quad b$ & $100,00 \mathrm{a}$ & $57,50 \quad \mathrm{~b}$ & $2,07 \quad \mathrm{c}$ \\
\hline Mediana & 68,75 & 53,75 a & 13,99 & $100,00 \mathrm{a}$ & $75,00 \mathrm{a}$ & $6,12 \mathrm{a}$ \\
\hline Apical & 50,67 & $42,67 \mathrm{a}$ & 26,79 a & $100,00 \mathrm{a}$ & 71,33 a & $4,62 \quad b$ \\
\hline $\mathrm{CV}(\%)$ & 28,95 & 18,19 & 27,18 & 0 & 13,95 & 22,59 \\
\hline
\end{tabular}

* Médias seguidas pela mesma letra na coluna não diferem significamente entre si, pelo teste Tukey, ao nível de 5\% de probabilidade.

TABELA 2 -Porcentagem de alporques calejados, enraizados e número médio de raízes de alporques de figueira 'Roxo de Valinhos' (Ficus carica L.), submetidos a diferentes ferimentos no ramo, e porcentagem de alporques vivos, brotados e número médio de brotos após 30 dias do transplantio. Marechal Cândido Rondon-PR, UNIOESTE, 2007.

\begin{tabular}{|c|c|c|c|c|c|c|}
\hline \multirow{2}{*}{$\begin{array}{c}\text { Ferimento } \\
\text { no ramo }\end{array}$} & \multicolumn{3}{|c|}{ Variáveis analisadas aos 60 dias* } & \multicolumn{3}{|c|}{ Variáveis analisadas após transplantio* } \\
\hline & $\begin{array}{c}\% \text { Alporques } \\
\text { calejados }\end{array}$ & $\begin{array}{c}\text { \% Alporques } \\
\text { enraizados }\end{array}$ & $\begin{array}{c}\mathrm{N}^{\circ} \text { raízes por } \\
\text { alporque }\end{array}$ & $\begin{array}{c}\% \text { Alporques } \\
\text { vivos } \\
\end{array}$ & $\begin{array}{c}\% \text { Alporques } \\
\text { brotados }\end{array}$ & $\begin{array}{c}\mathrm{N}^{\circ} \text { médio } \\
\text { brotos }\end{array}$ \\
\hline Sem corte & 85,00 a & $60,00 \mathrm{a}$ & $18,58 \mathrm{a}$ & $100,00 \mathrm{a}$ & $73,33 \mathrm{ab}$ & $4,43 \quad b$ \\
\hline Um corte & 81,67 a & $50,00 \mathrm{a}$ & $12,50 \mathrm{a}$ & $100,00 \mathrm{a}$ & 80,00 a & 6,03 a \\
\hline Dois corte & 60,00 & 28,33 & $12,73 \mathrm{a}$ & $100,00 \mathrm{a}$ & 63,33 bc & $3,83 \quad b$ \\
\hline Anel & 45,00 & 37,27 & $25,12 \mathrm{a}$ & $100,00 \mathrm{a}$ & 53,63 & 2,63 \\
\hline $\mathrm{CV}(\%)$ & 28,95 & 18,19 & 27,18 & 0 & 13,95 & 22,59 \\
\hline
\end{tabular}

* Médias seguidas pela mesma letra na coluna não diferem significamente entre si, pelo teste Tukey, ao nível de 5\% de probabilidade. 
TABELA 3- Porcentagem de alporques calejados, enraizados e número médio de raízes de alporques de figueira 'Roxo de Valinhos' (Ficus carica L.), realizados com a utilização de diferentes substratos. Marechal Cândido Rondon-PR, UNIOESTE, 2007.

\begin{tabular}{|c|c|c|c|}
\hline \multirow[t]{2}{*}{ Substratos } & \multicolumn{3}{|c|}{ Variáveis analisadas* } \\
\hline & $\%$ Alporques calejados & $\begin{array}{c}\% \text { Alporques } \\
\text { enraizados }\end{array}$ & $\mathrm{N}^{\circ}$ raízes alporque \\
\hline Esfagno (E) & $100,00 \mathrm{a}$ & $28,12 \quad \mathrm{~b}$ & $2,62 \quad b$ \\
\hline Casca de pínus (CP) & 98,75 a & $46,00 \mathrm{a}$ & $5,84 \mathrm{a}$ \\
\hline $\mathrm{E}+\mathrm{CP}(1: 1)$ & 97,33 a & $39,00 \quad \mathrm{ab}$ & $3,54 \mathrm{ab}$ \\
\hline $\mathrm{Cv}(\%)$ & 6,33 & 20,38 & 23,63 \\
\hline
\end{tabular}

* Médias seguidas pela mesma letra na coluna não diferem significamente entre si, pelo teste Tukey, ao nível de 5\% de probabilidade.

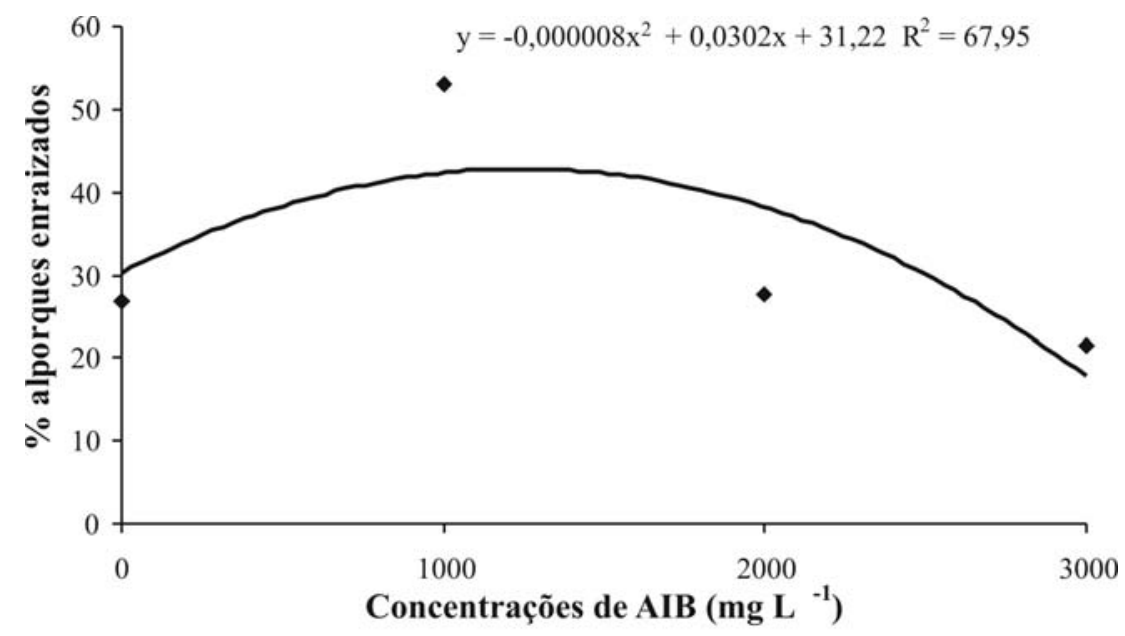

FIGURA 1- Porcentagem de alporques enraizados de figueira 'Roxo de Valinhos' submetidos a diferentes concentrações de AIB. Marechal Cândido Rondon-PR, UNIOESTE, 2007.

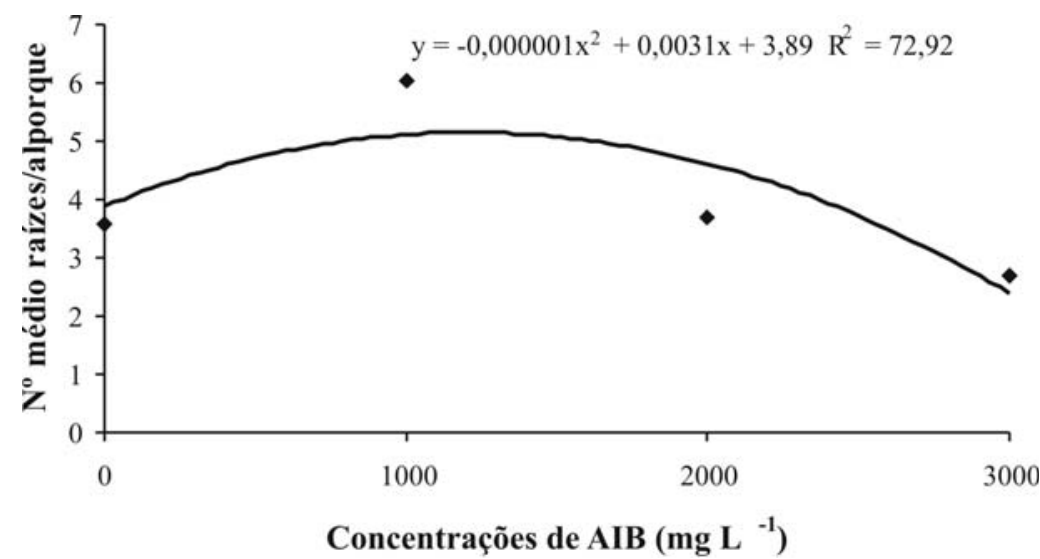

FIGURA 2 - Número médio de raízes por alporques de figueira 'Roxo de Valinhos' submetidos a diferentes concentrações de AIB. Marechal Cândido Rondon-PR, UNIOESTE, 2007. 


\section{REFERÊNCIAS}

ALVARENGA, A.A.; ABRAHAO, E.; FRAGUAS, J.C.; CARVALHO, V.L.; SILVA, R.A.; SANTA CECILIA, L.V.C.; CUNHA, R.L.; SILVA, V.J. Figo (Ficus carica L.). In: TRAZILBO, J.P.Jr.; MADELAINE V. (Org.). 101 Culturas: manual de tecnologias agrícolas. Belo Horizonte: EPAMIG, 2007. p.365-372.

CASTRO, L.A.S.; SILVEIRA, C.A P. Propagação vegetativa do pessegueiro por alporquia. Revista Brasileira de Fruticultura, Jaboticabal, v. 25, n. 2, p. 368-370, 2003.

CHALFUN, N.N.J.; PASQUAL, M.; HOFFMANN, A. Fruticultura comercial: frutíferas de clima temperado. Lavras: UFLA/FAEPE, 1997. 304p.

CHALFUN, N.N.J.; ABRAHÃO, E.; ALVARENGA, A.A.; REGINA, M.A.; PIO, R. Poda e condução da figueira. Lavras: UFLA, 2002. 12p. (Boletim Técnico, 104).

FACHINELLO, J.C.; HOFFMANN, A.; NACHTIGAL, J.C. Propagação de plantas frutíferas. Brasília: Embrapa, 2005.221p.

FERREIRA, D.F. Análise estatística por meio do SISVAR (Sistema para Análise de Variância) para Windows versão 4.0. In: REUNIÃO ANUAL DA REGIÃO BRASILEIRA DA SOCIEDADE INTERNACIONAL DE BIOMETRIA, 45., 2000, São Carlos. Anais... São Carlos: UFSCar, 2000. p.255-258.

GOMES, F.P. Curso de estatística experimental. 14. ed. Piracicaba: USP/ESALQ, 2000. 477p.

GONÇALVES, F.C.; CHALFUN, N.N.J.; ALVARENGA, A.A.; MIRANDA, C.S. Influência da forma de acondicionamento sob frio na sobrevivência de mudas de figueira. Ciência e Agrotecnologia, Lavras, v.27, n.4, p.798-803, 2003.
HARTMANN, H.T.; KESTER, D.E.; DAVIES JUNIOR, F.T.; GENEVE, R.L. Plant propagation: principles and practices. $7^{\text {th }}$ ed. New Jersey: Prentice Hall, 2002. 880p.

NORBERTO, P.M.; CHALFUN, N.N.J.; PASQUAL, M.; VEIGA, R.D.; PEREIRA, G.E.; MOTA, J.H. Efeito da época de estaquia e do AIB no enraizamento de estacas de figueira (Ficus carica L.). Ciência e Agrotecnologia, Lavras, v. 25, n. 3, p. 533-541, 2001.

PIO, R. Ácido indolbutírico e sacarose no enraizamento de estacas apicais e desenvolvimento inicial da figueira (Ficus carica L.). 2002. 109 f. Dissertação (Mestrado em Agronomia) Universidade Federal de Lavras, Lavras, 2002.

PIO, R.; RAMOS, J.D.; CHALFUN, N.N.J.; COELHO, J.H.C.; GONTIJO, T.C.A.; CARRIJO, E.P.; VILLA, F. Enraizamento adventícios de estacas apicais de figueira e desenvolvimento inicial das plantas no campo. Ciência e Agrotecnologia, Lavras, v.28, n.1, p.215-221, 2004.

PIO, R.; RAMOS, J.D.; CHALFUN, N.N.J.; GONTIJO, T.C.A.; MENDONÇA, V.; CARRIJO, E.P.; CHAGAS, E.A. Propagação de estacas apicais de figueira: diferentes ambientes, ácido indolbutírico e tipo de estaca. Ciência e Agrotecnologia, Lavras, v.30, n.5, p.1021-1026, 2006a.

PIO, R.; CHAGAS, E.A.; BARBOSA, W.; MENDONÇA, V.; SCARPARE FILHO, J.A.; GUIMARAES, V.F. Enraizamentos de estacas de figueira coletadas em diferentes posições no ramo e ambientes de propagação distintos. Scientia Agraria Paranaensis, Cascavel, v.5, p.1-5, 2006b.

SMIDERLE, O.S.; MINAMI, K. Emergência e vigor de plântulas de goiabeira em diferentes substratos. Revista Científica Rural, Bagé, v.6, n.1,p.38-45, 2001. 